\title{
Gyrodactylus magadiensis n. sp. (Monogenea, Gyrodactylidae) parasitising the gills of Alcolapia grahami (Perciformes, Cichlidae), a fish inhabiting the extreme environment of Lake Magadi, Kenya
}

\author{
Quinton Marco Dos Santos, John Ndegwa Maina, and Annemariè Avenant-Oldewage* \\ Department of Zoology, University of Johannesburg, PO Box 524, Auckland Park, 2006 Johannesburg, South Africa
}

Received 31 July 2019, Accepted 6 December 2019, Published online 20 December 2019

\begin{abstract}
A new species of Gyrodactylus von Nordmann, 1832 is described from the gills of Alcolapia grahami, a tilapian fish endemic to Lake Magadi. This alkaline soda lake in the Rift Valley in Kenya is an extreme environment with $\mathrm{pH}$ as high as 11 , temperatures up to $42{ }^{\circ} \mathrm{C}$, and diurnal fluctuation between hyperoxia and virtual anoxia. Nevertheless, gyrodactylid monogeneans able to survive these hostile conditions were detected from the gills the Magadi tilapia. The worms were studied using light microscopy, isolated sclerites observed using scanning electron microscopy, and molecular techniques used to genetically characterize the specimens. The gyrodactylid was described as Gyrodactylus magadiensis n. sp. and could be distinguished from other Gyrodactylus species infecting African cichlid fish based on the comparatively long and narrow hamuli, a ventral bar with small rounded anterolateral processes and a tongue-shaped posterior membrane, and marginal hooks with slender sickles which are angled forward, a trapezoid to square toe, rounded heel, a long bridge prior to reaching marginal sickle shaft, and a long lateral edge of the toe. The species is also distinct from all other Gyrodactylus taxa based on the ITS region of rDNA (ITS1-5.8s-ITS2), strongly supporting the designation of a new species. These findings represent the second record of Gyrodactylus from Kenya, with the description of G. magadiensis bringing the total number of Gyrodactylus species described from African cichlids to 18.
\end{abstract}

Key words: Lake Magadi, Alcolapia grahami, Gyrodactylus, Kenya, Soda Lake.

Résumé - Gyrodactylus magadiensis n. sp. (Monogenea, Gyrodactylidae), parasite des branchies d'Alcolapia grahami (Perciformes, Cichlidae), un poisson habitant l'environnement extrême du lac Magadi au Kenya. Une nouvelle espèce de Gyrodactylus von Nordmann, 1832 est décrite à partir des branchies d'Alcolapia grahami, un tilapia endémique du lac Magadi. Ce lac de soude alcaline dans la vallée du Rift au Kenya est un environnement extrême avec un $\mathrm{pH}$ allant jusqu'à 11 , des températures allant jusqu'à $42{ }^{\circ} \mathrm{C}$ et des fluctuations diurnes entre l'hyperoxie et l'anoxie virtuelle. Néanmoins, des Monogènes Gyrodactylidae capables de survivre dans ces conditions hostiles ont été détectés sur les branchies du tilapia de Magadi. Les vers ont été étudiés par microscopie optique, les sclérites isolés ont été observés au microscope électronique à balayage et des techniques moléculaires ont été utilisées pour caractériser génétiquement les spécimens. Le gyrodactylidé est décrit comme étant Gyrodactylus magadiensis n. sp. et se distingue des autres espèces de Gyrodactylus infectant les cichlidés d'Afrique grâce à ses hamuli relativement longs et étroits, à une barre ventrale avec de petits processus antérolatéraux arrondis et à une membrane postérieure en forme de langue, ainsi qu'à des crochets marginaux à faucilles minces inclinées vers l'avant, un trapèze à bout carré, un talon arrondi, un long pont avant d'atteindre la faucille marginale et un long bord latéral de l'extrémité. L'espèce est également distincte de tous les autres taxons de Gyrodactylus sur la base sur la région ITS de 1'ADNr (ITS1-5.8s-ITS2), ce qui soutient fortement la désignation d'une nouvelle espèce. Ces découvertes représentent la seconde mention d'un Gyrodactylus au Kenya, et la description de G. magadiensis amène à 18 le nombre total d'espèces de Gyrodactylus décrites parmi les cichlidés d'Afrique.

\section{Introduction}

Fishes of the genus Alcolapia Thys van den Audenaerde, 1969 are African cichlids that occur in two of the most severe

*Corresponding author: aoldewage@uj.ac.za environments in the eastern African Rift Valley, namely the Soda Lakes Magadi and Natron, located in Kenya and Tanzania, respectively $[53,67]$. These two lakes were once a continuous water body, separated $\sim 10,000$ years ago $[7,14$, 22, 52]. Fish subsist in scattered lagoons and sites at the periphery of Lake Magadi where particularly extreme conditions 
occur, which include $\mathrm{pH}$ up to 11 , titration alkalinity $>300 \mathrm{mM}$, osmolarity $525 \mathrm{mOsm}$, temperatures as high as $42{ }^{\circ} \mathrm{C}$, and $\mathrm{O}_{2}$ levels fluctuating diurnally between hyperoxia during the day and virtual anoxia at night $[5,28,39,41,46,53,56,61,66$, $68,69]$. Being a closed lake, the alkaline lagoons are recharged by hot geothermal springs where temperatures can reach as high as $86{ }^{\circ} \mathrm{C}$. Despite these hostile conditions, a single species of cichlid fish, Alcolapia grahami (Boulenger, 1912), or the Magadi tilapia, inhabits the hot and highly alkaline waters of this lake $[41,62]$. The Magadi tilapia has developed exceptional morphological and physiological adaptations, especially to cope with the high $\mathrm{pH}$, extreme alkalinity, and the shifting oxygen availability. These include: (a) excretion of nitrogenous waste in the form of urea instead of ammonia through the ornithineurea cycle [69], (b) an atypically thin blood-water barrier in the gills $[38,40]$, and (c) the use of the swim bladder as a primitive air-breathing organ [40].

Gills of African cichlids are often infected by monogenean parasites of the genera Gyrodactylus von Nordmann, 1832 and Cichlidogyrus Paperna, 1960. Using the conventional estimate of, on average, 1 monogenean species per fish host, the projected international diversity of the genus Gyrodactylus was proposed to be 20,000 species [4, 24]. Nevertheless, the number of Gyrodactylus spp. from African freshwater fish amounts to 35 [57], of which only 17 are from cichlids (Table 1). Thus, the species currently known denote only a fragment of the number of anticipated Gyrodactylus spp. in Africa, especially from cichlids. In contrast, even though Gyrodactylus is predicted to be more specious, substantially more species of Cichlidogyrus have been described from African cichlids (122 according to Řehulková et al. [57]). Within Gyrodactylus, several morphological criteria are used to discriminate between species. These include metrics of haptoral sclerites, the form and shape of marginal hooks, the presence of additional haptoral elements (e.g. ventral bar processes), the number and arrangement of the MCO spines, and the morphology, and shape of the ventral bar membrane (e.g. [18, 24, 64]). The use of molecular approaches to support taxonomic studies of these parasites have also been implemented in the past few decades, but genetic data for several species are not yet available. Regarding the 17 species of Gyrodactylus from cichlids, for example, sequence data for only 13 are currently available. Only a single Gyrodactylus species has been described from Kenya, Gyrodactylus malalai Přikrylová, Blažek and Gelnar, 2012 from Oreochromis niloticus niloticus (L.) and Tilapia zillii (Gervais) in Lake Turkana.

Monogenean parasites have previously been recorded from systems with extreme physicochemical environments, such as Gyrodactylus salinae Paladini, Huyse and Shinn 2011 from the hypersaline Cervia Saline in Italy [45], and as such may occur alongside the highly adapted Magadi tilapia. Specimens of A. grahami collected at the Fish Springs Lagoon of Lake Magadi provided a unique opportunity to study this species for possible parasite infections. In doing so, an unidentified species of Gyrodactylus, adapted to, and thriving, in these extreme conditions, was detected. Thus, this paper represents the morphological and molecular description of a new species of Gyrodactylus, and the first record of a gyrodactylid parasite from the gills of the Lake Magadi tilapia, A. grahami from Kenya.

\section{Materials and methods Collection}

Fish were collected (permit number NCST/RRI/12/1/ MAS/99) from the Fish Spring Lagoon of Lake Magadi (Fig. 1B and C) in July 2013 and June 2018. Fish were specifically collected from the most peripheral pool (1 in Fig. 1C). Fish were euthanised on site by severing the spinal cord and the whole fish preserved in either formalin (July 2013) or absolute ethanol (June 2018). At the University of Johannesburg, gills were removed from the preserved fish and studied for the presence of parasites, with attached worms removed from the gills using fine dissecting needles and detached worms picked carefully from the fixative.

\section{Morphometric analyses}

Formalin fixed worms were washed in water, dehydrated in a series of ethanol $(30 \%, 50 \%$, and $70 \%$ ethanol), and subsequently mounted and cleared in glycerine ammonium picrate (GAP) [40] for examination of the haptoral sclerites and male copulatory organ (MCO). Some specimens were also stained with Horen's trichrome [41] and cleared and mounted in lactophenol. Light microscopy, using both phase and differential interference contrast approaches, were used to study the shape and dimensions of sclerotized structures using a Zeiss Axioplan 2 imaging light microscope with Axiovision 4.7.2 software (Carl Zeiss, Jena, Switzerland). Micrographs were used to draw taxonomically important structures. The measurements of hamuli and other haptoral sclerites (28 point-to-point measurements) were taken according to Shinn et al. [60] and GarcíaVásquez et al. [15], and the measurements of the parasite's whole body were taken according to Christison et al. [8]. All measurements are in micrometres unless stated otherwise, and presented as a mean and range in parentheses. Measurements of the haptoral sclerites are presented in full alongside the only other Gyrodactylus species described from Kenya (G. malalai) in Table 2. After analyses, worms were removed from the slides, dehydrated through a graded ethanol series, and mounted in Canada balsam for permanent storage [11].

Due to the fixation of samples in formalin and high concentration ethanol, SEM of whole worms did not produce viable results. To study the sclerites at higher magnification, worms were digested on a concavity slide using the digestion buffer from a DNA extraction kit, digested tissue removed, rinsed, and prepared for SEM [35, 42, 58-60]. Slides were gold sputter coated using an Emscope SC500 Sputter Coater (Quorum Technologies, Newhaven, UK) and studied with using a Vega 3 LMH scanning electron microscope (Tescan, Brno, Czech Republic) at $3.4 \mathrm{kV}$.

\section{Molecular characterisation}

For genomic identification, 10 ethanol fixed specimens were rehydrated, digested, and genomic DNA extracted using a NucleoSpin ${ }^{\circledR}$ Tissue kit (Macherey-Nagel, Düren, Germany), following the manufacturer's protocols. The region of rDNA spanning the $3^{\prime}$ end of $18 \mathrm{~S}$, ITS1, 5.8S, ITS2, to the $5^{\prime}$ end of $28 \mathrm{~S}$ was targeted using primers BD1 $\left(5^{\prime}-\mathrm{GTC}\right.$ GTA ACA 
Table 1. Collection details, including author, host, and distribution, for all species of Gyrodactylus von Nordmann, 1832 described and recorded from African cichlids. Type hosts in bold.

\begin{tabular}{|c|c|c|c|c|}
\hline Species & Authors & Host & Country & Reference \\
\hline G. aegypticus* & $\begin{array}{l}\text { El-Naggar and El-Tantawy, } \\
2003\end{array}$ & Coptodon zillii (Gervais) & Egypt & {$[10]$} \\
\hline G. chitandiri & $\begin{array}{l}\text { Zahradníčková, Barson, } \\
\text { Luus-Powell and } \\
\text { Přikrylová, } 2016\end{array}$ & $\begin{array}{l}\text { Coptodon rendalli (Boulenger) } \\
\text { Pseudocrenilabrus philander (Weber) }\end{array}$ & $\begin{array}{l}\text { Chirundu, Zambezi River, and } \\
\text { Lake Kariba, Zimbabwe }\end{array}$ & {$[70]$} \\
\hline \multirow[t]{9}{*}{ G. cichlidarum } & Paperna, 1968 & Sarotherodon galilaeus (L.) & $\begin{array}{l}\text { Accra Plain and Akuse Lagoon, } \\
\text { Ghana }\end{array}$ & [47] \\
\hline & & $\begin{array}{l}\text { Hemichromis fasciatus } \text { Peters } \\
\text { Hemichromis bimaculatus Gill } \\
\text { Coptodon zillii (Gervais) }\end{array}$ & $\begin{array}{l}\text { Accra Plain and Akuse Lagoon, } \\
\text { Ghana }\end{array}$ & [47] \\
\hline & & $\begin{array}{l}\text { Sarotherodon galilaeus (L.) } \\
\text { Sarotherodon melanotheron Rüppell } \\
\text { Coptodon guineensis (Günther) } \\
\text { Coptodon zillii (Gervais) }\end{array}$ & $\begin{array}{l}\text { Accra Plain and Akuse Lagoon, } \\
\text { Ghana }\end{array}$ & [49] \\
\hline & & Hemichromis fasciatus Peters & $\begin{array}{l}\text { Mare Simenti, Niokolo Koba } \\
\text { National Park, Senegal }\end{array}$ & {$[52]$} \\
\hline & & Oreochromis niloticus (L.) & $\begin{array}{l}\text { Cultured stock, University of } \\
\text { Stirling, UK }\end{array}$ & [13] \\
\hline & & Astronotus ocellatus (Agassiz) & Various pet stores, Tehran, Iran & [43] \\
\hline & & $\begin{array}{l}\text { Poecilia mexicana Steindachner } \\
\text { Poeciliopsis gracilis (Heckel) } \\
\text { Pseudoxiphophorus bimaculatus } \\
\quad \text { (Heckel) }\end{array}$ & Puebla and Michoacán, Mexico & {$[17]$} \\
\hline & & Oreochromis niloticus (L.) & $\begin{array}{l}\text { University of the Philippines, } \\
\text { Visayas, Iloilo Province, } \\
\text { Philippines }\end{array}$ & [9] \\
\hline & & Oreochromis niloticus (L.) & Vietnam Mekong River Delta & {$[2]$} \\
\hline G. ergensi & $\begin{array}{l}\text { Přikrylová, Matějusová, } \\
\text { Musilová and Gelnar, } 2009\end{array}$ & $\begin{array}{l}\text { Sarotherodon galilaeus (L.) } \\
\text { Oreochromis niloticus (L.) }\end{array}$ & $\begin{array}{l}\text { Mare Simenti, Niokolo Koba } \\
\text { National Park, Senegal }\end{array}$ & {$[52]$} \\
\hline G. haplochromi & Paperna, 1973 & $\begin{array}{l}\text { Haplochromis angustifrons } \\
\text { Boulenger }\end{array}$ & Lake George, Uganda & [48] \\
\hline G. hildae & $\begin{array}{l}\text { García-Vásquez, Hansen, } \\
\text { Christison, Bron, and } \\
\text { Shinn, } 2011\end{array}$ & Oreochromis niloticus (L.) & $\begin{array}{l}\text { Tributary of the Baro River, } \\
\text { Gambela, Ethiopia }\end{array}$ & [14] \\
\hline \multirow[t]{2}{*}{ G. malalai } & $\begin{array}{l}\text { Přikrylová, Blažek, and } \\
\text { Gelnar, } 2012\end{array}$ & $\begin{array}{l}\text { Oreochromis niloticus (L.) } \\
\text { Coptodon zillii (Gervais) }\end{array}$ & Lake Turkana, Kenya & {$[53]$} \\
\hline & & Oreochromis niloticus (L.) & Blue Nile, Sudan & {$[53]$} \\
\hline \multirow[t]{3}{*}{ G. nyanzae } & Paperna, 1973 & $\begin{array}{l}\text { Oreochromis variabilis (Boulenger) } \\
\text { Oreochromis niloticus }(\mathrm{L} .) \mathrm{X} \\
\text { Oreochromis mweruensis Trewavas } \\
\text { Coptodon rendalli (Boulenger) }\end{array}$ & $\begin{array}{l}\text { Lake Victoria, Uganda } \\
\text { Kipopo station, Haut-Katanga } \\
\text { province, DRC }\end{array}$ & {$[48]$} \\
\hline & & Oreochromis niloticus (L.) & $\begin{array}{l}\text { Chirundu, Zambezi River, } \\
\text { Zimbabwe }\end{array}$ & [70] \\
\hline & & $\begin{array}{l}\text { Oreochromis mweruensis Trewavas } \\
\text { Coptodon rendalli (Boulenger) }\end{array}$ & $\begin{array}{l}\text { Kipopo and Luapula River, } \\
\text { Bangweulu-Mweru, DRC }\end{array}$ & {$[27]$} \\
\hline G. occupatus & $\begin{array}{l}\text { Zahradníčková, Barson, } \\
\text { Luus-Powell and } \\
\text { Přikrylová, } 2016\end{array}$ & $\begin{array}{l}\text { Oreochromis niloticus } \text { (L.) } \\
\text { Pharyngochromis acuticeps } \\
\quad \text { (Steindachner) } \\
\text { Pseudocrenilabrus philander (Weber) }\end{array}$ & Lake Chivero, Zimbabwe & [70] \\
\hline G. parisellei & $\begin{array}{l}\text { Zahradníčková, Barson, } \\
\text { Luus-Powell and } \\
\text { Přikrylová, } 2016\end{array}$ & $\begin{array}{l}\text { Oreochromis niloticus (L.) } \\
\text { Pseudocrenilabrus philander (Weber) }\end{array}$ & $\begin{array}{l}\text { Lake Chivero and Lake Kariba, } \\
\text { Zimbabwe }\end{array}$ & [70] \\
\hline G. shariffi & $\begin{array}{l}\text { Cone, Arthur and Bondad- } \\
\text { Reantaso, } 1995\end{array}$ & Oreochromis niloticus (L.) & $\begin{array}{l}\text { University of the Philippines, } \\
\text { Visayas, nIloilo Province, } \\
\text { Philippines }\end{array}$ & [9] \\
\hline \multirow[t]{2}{*}{ G. sturmbaueri } & $\begin{array}{l}\text { Vanhove, Snoeks, Volckaert } \\
\text { and Huyse, } 2011\end{array}$ & Simochromis diagramma (Günther) & $\begin{array}{l}\text { Kalambo Lodge, Lake } \\
\text { Tanganyika, Zambia }\end{array}$ & {$[64]$} \\
\hline & & Pseudocrenilabrus philander (Weber) & $\begin{array}{l}\text { Chirundu, Zambezi River, } \\
\text { Zimbabwe }\end{array}$ & [70] \\
\hline
\end{tabular}


Table 1. (Continued)

\begin{tabular}{|c|c|c|c|c|}
\hline Species & Authors & Host & Country & Reference \\
\hline \multirow[t]{2}{*}{ G. thlapi } & \multirow[t]{2}{*}{$\begin{array}{l}\text { Christison, Shinn and van As, } \\
2005\end{array}$} & Pseudocrenilabrus philander (Weber) & $\begin{array}{l}\text { Shakawe, Sepopa and Seronga, } \\
\text { Okavango Delta, Botswana }\end{array}$ & {$[8]$} \\
\hline & & Pseudocrenilabrus philander (Weber) & Baberspan Wetland, South Africa & [63] \\
\hline G. thysi & $\begin{array}{l}\text { Vanhove, Snoeks, Volckaert } \\
\text { and Huyse, } 2011\end{array}$ & Simochromis diagramma (Günther) & $\begin{array}{l}\text { Kalambo Lodge, Lake } \\
\text { Tanganyika, Zambia }\end{array}$ & {$[64]$} \\
\hline G. ulinganisus & $\begin{array}{l}\text { García-Vásquez, Hansen, } \\
\text { Christison, Bron and } \\
\text { Shinn, } 2011\end{array}$ & Oreochromis mossambicus (Peters) & $\begin{array}{l}\text { Welgevallen, Stellenbosch, } \\
\text { South Africa }\end{array}$ & {$[14]$} \\
\hline \multirow[t]{2}{*}{ G. yacatli } & \multirow[t]{2}{*}{$\begin{array}{l}\text { García-Vásquez, Hansen, } \\
\text { Christison, Bron and } \\
\text { Shinn, } 2011\end{array}$} & Oreochromis niloticus (L.) & $\begin{array}{l}\text { Gania de Pucté, Municipal de } \\
\text { Chablé, Tabasco, Mexico } \\
\text { Merida, Mexico } \\
\text { Culiacan, Mexico }\end{array}$ & [14] \\
\hline & & $\begin{array}{l}\text { Oreochromis niloticus (L.) } \\
\text { Pseudocrenilabrus philander (Weber) }\end{array}$ & $\begin{array}{l}\text { Chirundu, Zambezi River, } \\
\text { Zimbabwe }\end{array}$ & {$[70]$} \\
\hline G. zimbae & $\begin{array}{l}\text { Vanhove, Snoeks, Volckaert } \\
\text { and Huyse, } 2011\end{array}$ & $\begin{array}{l}\text { Simochromis diagramma (Günther) } \\
\text { Ctenochromis horei (Günther) }\end{array}$ & $\begin{array}{l}\text { Kalambo Lodge, Lake } \\
\text { Tanganyika, Zambia }\end{array}$ & {$[64]$} \\
\hline
\end{tabular}

* Nomen nudum.

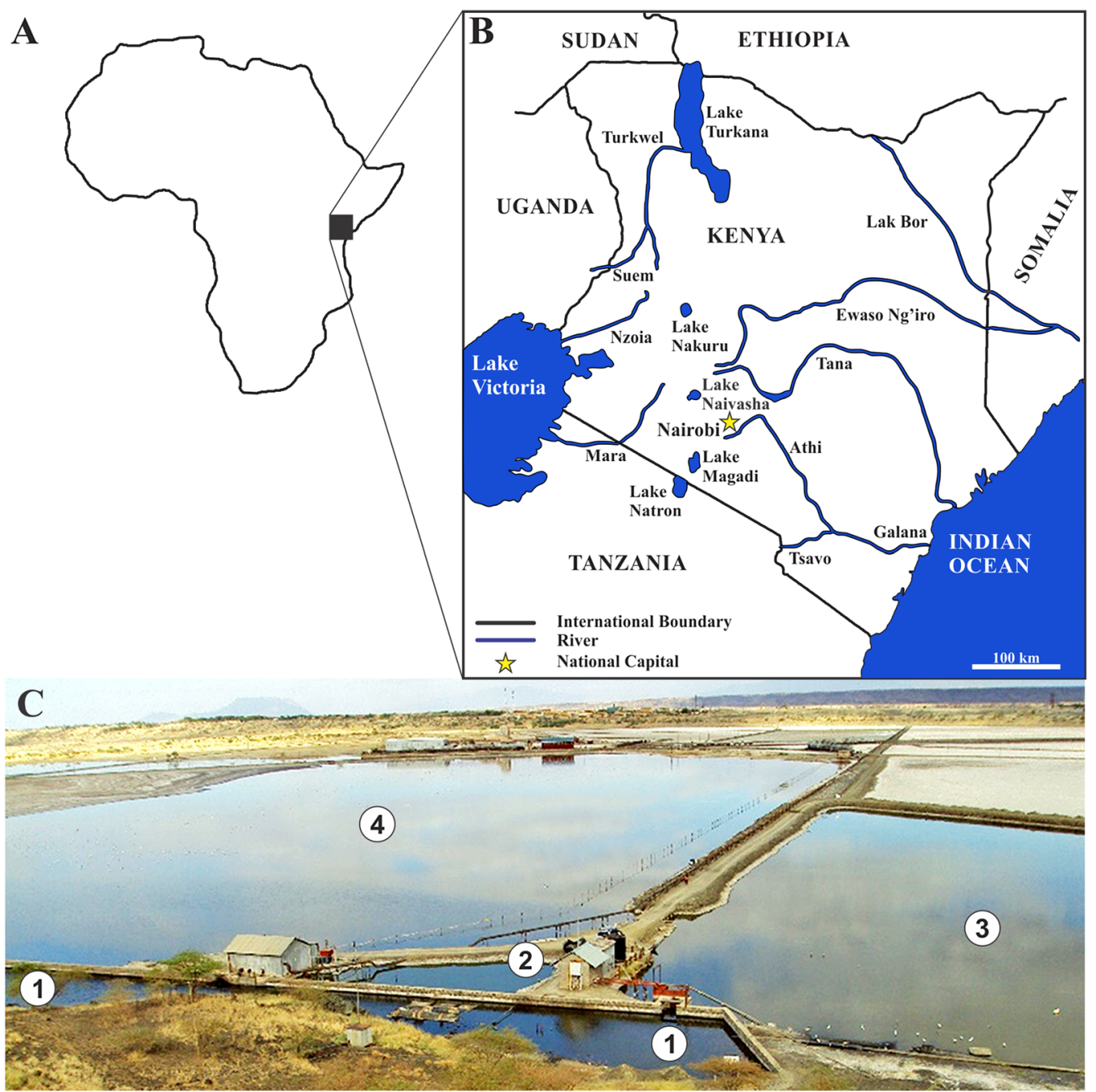

Figure 1. Collection sites from which Alcolapia grahami were collected. (A) Silhouette of Africa showing area of study; (B) map of study area indicating countries, water bodies, and relation of Lake Magadi to Nairobi; (C) fish spring lagoon of Lake Magadi from which the fish specimens were collected. 
Table 2. Morphological measurements of Gyrodactylus magadiensis n. sp. from the Magadi tilapia, Alcolapia grahami collected from Lake Magadi, Kenya. Data are presented alongside data for the only other previously described Gyrodactylus species from Kenya. Data compiled from previous study directly transposed as noted by author.

\begin{tabular}{|c|c|c|}
\hline \multirow[t]{3}{*}{ Measurement } & G. magadiensis n. sp. & G. malalai \\
\hline & $n=24$ & $n=18$ \\
\hline & Present study & Přikrylová et al. [53] \\
\hline Total body length & $267 \pm 53(202-387)$ & $792 \pm 74.8(666-876)$ \\
\hline Total body width & $65 \pm 17.6(41.4-100)$ & $118 \pm 13.7(98-136)$ \\
\hline Pharynx length & $21.1 \pm 4.6(13.8-29.7)$ & $42.5 \pm 6.2(34-49.5)$ \\
\hline Pharynx width & $19.7 \pm 4(13.6-28.2)$ & $37.2 \pm 6.4(26.5-44)$ \\
\hline Posterior pharynx length & $15.6 \pm 2(13.5-18)$ & \\
\hline Posterior pharynx width & $13.2 \pm 2.2(11-15)$ & \\
\hline MCO length & $11.3 \pm 1.9(9.6-14)$ & \\
\hline MCO width & $8.5 \pm 1.3(6.9-10.2)$ & \\
\hline MCO spines & $1 \mathrm{~L}, 6 \mathrm{~S}$ & $1 \mathrm{~L}, 4-5 \mathrm{~S}$ \\
\hline \multicolumn{3}{|l|}{ Hamulus } \\
\hline Total length & $62.8 \pm 5.6(53.6-73.7)$ & $109 \pm 3.9(102-116.5)$ \\
\hline Aperture & $31 \pm 2.2(27.3-35.3)$ & \\
\hline Point shaft width & $6.9 \pm 0.9(5.3-8.7)$ & \\
\hline Point length & $24.2 \pm 2.4(19.9-29.5)$ & $40.2 \pm 2.9(36-49)$ \\
\hline Distal shaft width & $3.5 \pm 0.5(2.6-4.6)$ & \\
\hline Shaft length & $38.7 \pm 2.2(34.9-43.6)$ & $74.5 \pm 2.8(68.5-78)$ \\
\hline Inner curve length & $24.9 \pm 2.3(21.4-30.2)$ & \\
\hline Aperture angle & $60 \pm 7.4(50.1-78.1)$ & \\
\hline Point curve angle & $23.7 \pm 5.4(11.1-36.4)$ & \\
\hline Inner aperture angle & $66.8 \pm 8.5(55.9-85.5)$ & \\
\hline Root length & $22.3 \pm 3.4(16.9-29.9)$ & $45 \pm 5.5(32.5-54)$ \\
\hline \multicolumn{3}{|l|}{ Ventral bar } \\
\hline Length & $27.5 \pm 2.7(22.6-33.3)$ & \\
\hline Width & $20.8 \pm 3.5(14.6-26.4)$ & $16 \pm 1.5(23.5-28.5)$ \\
\hline Process to mid-length & $3.5 \pm 0.9(2.1-5.1)$ & \\
\hline Mid-length & $5.2 \pm 1(3.5-7)$ & $8.3 \pm 0.6(7.5-9.5)$ \\
\hline Process length & $3 \pm 0.5(2-4)$ & \\
\hline Membrane length & $18.7 \pm 1.6(15.9-22.8)$ & $14.5 \pm 1.4(12.5-16.5)$ \\
\hline \multicolumn{3}{|l|}{ Dorsal bar } \\
\hline Length & $2.6 \pm 0.5(2.1-3.6)$ & $2.3 \pm 0.2(2-2.5)$ \\
\hline Width & $13 \pm 1.9(9.8-17.8)$ & $23.5 \pm 1.1(22.5-25)$ \\
\hline \multicolumn{3}{|l|}{ Marginal hook } \\
\hline Total length & $27.7 \pm 2.5(23.4-31)$ & $32 \pm 0.6(31.5-33)$ \\
\hline Shaft length & $23.1 \pm 2.3(18.9-26)$ & $23.5 \pm 0.7(23-24.5)$ \\
\hline Sickle length & $5.4 \pm 0.4(4.7-6.1)$ & $8.5 \pm 0.3(8-9)$ \\
\hline Sickle point width & $4 \pm 0.5(3-4.7)$ & $6 \pm 0.3(5.5-6.5)$ \\
\hline Toe length & $1.8 \pm 0.2(1.5-2.2)$ & \\
\hline Sickle distal width & $3.4 \pm 0.3(2.9-4)$ & $7.3 \pm 0.5(6.5-8)$ \\
\hline Aperture & $4.4 \pm 0.5(3.6-5.1)$ & $8 \pm 0.5(7.5-9)$ \\
\hline Instep/arch height & $0.8 \pm 0.1(0.6-1)$ & \\
\hline Filament loop & $8 \pm 0.8(6.5-9.2)$ & \\
\hline
\end{tabular}

AGG TTT CCG TA-3') and BD2 (5'-TAT GCT TAA (G/A) TT CAG CGG GT-3') [34]. PCR was performed under the following conditions: initial denaturation at $94{ }^{\circ} \mathrm{C}$ for $5 \mathrm{~min}$, 30 cycles of $94{ }^{\circ} \mathrm{C}$ for $30 \mathrm{~s}, 56{ }^{\circ} \mathrm{C}$ for $30 \mathrm{~s}$, and $72{ }^{\circ} \mathrm{C}$ for $1 \mathrm{~min}$, followed by a final extension at $72{ }^{\circ} \mathrm{C}$ for $5 \mathrm{~min}$ as per Luo et al. [33]. Successful amplification was verified using $1 \%$ agarose gel impregnated with GelRed (Biotuim, Hayward, USA), and amplicons where sequenced using BigDye v3.1 chemistry (Applied Biosystems, Foster City, USA), following Avenant-Oldewage et al. [3]. Sequencing was performed on an ABI3730 automated sequencer (Applied Biosystems, Foster City, USA). Electropherograms were inspected and edited manually (if required) using Geneious R6 [30] and deposited in GenBank. Obtained sequences were aligned to sequence data for the 10 most closely related Gyrodactylus species as determined using BLASTn [1]. All sequences used (Table 3) were aligned using MAFFT [28, 29] via the EMBL-EBI portal. Aligned sequence data were analysed using MEGA 7 [32]. Genetic divergence among species of Gyrodactylus was estimated using uncorrected $p$-distances. Phylogenies were constructed with maximum likelihood (ML) methods, using the Tamura three-parameter model $(5$ categories $[+\mathrm{G}$, parameter $=0$. 3415]) (determined by the Model Selection tool in MEGA 7) as implemented in MEGA 7 [32]. Analyses were subject to 1000 bootstrap replicate variance estimation. Bayesian inference (BI) analyses were performed with BEAST v2.5.0 [6], using 
Table 3. List of Gyrodactylus species included in the phylogenetic analyses with their hosts, collection site, GenBank accession number, and reference.

\begin{tabular}{|c|c|c|c|c|}
\hline Species & Host & Locality & GenBank & Reference \\
\hline Gyrodactylus branchicus & Gasterosteus aculeatus & Bothnian Bay, Oulu, Finland & AY061977 & [71] \\
\hline Gyrodactylus branchicus & Gasterosteus aculeatus & Schelde River, Doel, Belgium & AF156669 & [72] \\
\hline Gyrodactylus rarus & Pungitius pungitius & Bothnian Bay, Oulu, Finland & AY061976 & [71] \\
\hline Gyrodactylus rarus & Pungitius pungitius & Wilkojadka River, Baltic Sea basin, Poland & FJ435193 & [56] \\
\hline Gyrodactylus rarus & Spinachia spinachia & Trondheim Biological, Station, Norway & AY338445 & [25] \\
\hline Gyrodactylus perlucidus & Zoarces viviparus & Manndalselva River, Barents Sea basin, Norway & FJ435202 & [56] \\
\hline Gyrodactylus medaka & Oryzias latipes & Midori River, Kumamoto, Japan & LC368478 & [45] \\
\hline Gyrodactylus medaka & Oryzias latipes & Nuta River, Hiroshima, Japan & LC368475 & [45] \\
\hline Gyrodactylus medaka & Oryzias latipes & AORI Laboratories, University of Tokyo, Tokyo & LC368479 & [45] \\
\hline Gyrodactylus medaka & Oryzias latipes & Sonose River, Tokushima, Japan & LC368477 & [45] \\
\hline Gyrodactylus alexgusevi & Lota lota & Oulujoki, Oulu, Finland & AY061979 & [71] \\
\hline Gyrodactylus groenlandicus & Myoxocephalus scorpius & Little Tancook Island, Nova Scotia, Canada & KJ095104 & [31] \\
\hline Gyrodactylus alexanderi & Gasterosteus aculeatus & Lake Skagvatn, South Trondelag County, Norway & JN695633 & [21] \\
\hline Gyrodactylus alexanderi & Gasterosteus aculeatus & Nanaimo, British Columbia, Canada & JF836144 & [19] \\
\hline Gyrodactylus alexanderi & Gasterosteus aculeatus & Horne Lake, Strait of Georgia, Pacific Ocean basin, Canada & FJ435201 & [56] \\
\hline Gyrodactylus wilkesi & Trematomus bernacchi & Prince Gustav Channel, Weddell Sea, Antarctica & LT719091 & [23] \\
\hline Gyrodactylus lamothei & Girardinichthys multiradiatus & San Nicolas Peralta, Lerma river basin, Mexico & KX555668 & [57] \\
\hline Gyrodactylus lamothei & Girardinichthys multiradiatus & San Nicolas Peralta, Lerma river basin, Mexico & KX555667 & [57] \\
\hline Gyrodactylus lamothei & Girardinichthys multiradiatus & San Nicolas Peralta, Lerma river basin, Mexico & KX555666 & [57] \\
\hline Gyrodactylus katamba & Goodea atripinnis & Santiago Mezquititlan, Queretaro, Mexico & KR815854 & [16] \\
\hline
\end{tabular}

10 million Markov chain Monte Carlo (MCMC) generations. BI data was prepared using BEAUTi 2, BEAST results assessed using TRACER v1.7.1, and trees processed using TreeAnnotator v2.5.0 and edited using FigTree v1.4.3.

\section{Results}

\section{Gyrodactylus magadiensis n. sp.}

urn:lsid:zoobank.org:act:89E29993-EC14-4AA3-AAE2C569D91B5FBD

Type host: Alcolapia grahami (Boulenger, 1912) (Perciformes, Cichlidae)

Type locality: Lake Magadi, Eastern Rift Valley, Kenya $\left(1^{\circ} 53^{\prime} 28.4^{\prime \prime} \mathrm{S}, 36^{\circ} 18^{\prime} 09.6^{\prime \prime} \mathrm{E}\right)$

Infection site: Gills

Type material: Holotype: Mounted in Canada Balsam and deposited in the Iziko South African Museum, Cape Town, South Africa (accession no. SAM - A091374). Paratypes: four specimens deposited in the Iziko South African Museum, Cape Town, South Africa (accession no. SAMC - A091375 to SAMC - A091378); four specimens deposited in the Natural History Museum, London, UK (accession nos. NHMUK 2019.12.6.1 to NHMUK 2019.12.6.4); and four specimens deposited in the Royal Museum for Central Africa in Tervuren, Belgium (accession nos. M.T.39080 to M.T.390803).

ITS rDNA sequences: Representative sequence submitted to GenBank (accession no. MN738699).

Etymology: The species is named after Lake Magadi from which the specimens were collected.

\section{Morphological description (Figs. 2 and 3, Table 3)}

Description based on 24 individuals. Specimens 267.1 (202-387.2) long, and 65 (41.4-100.1) wide at level of anterior
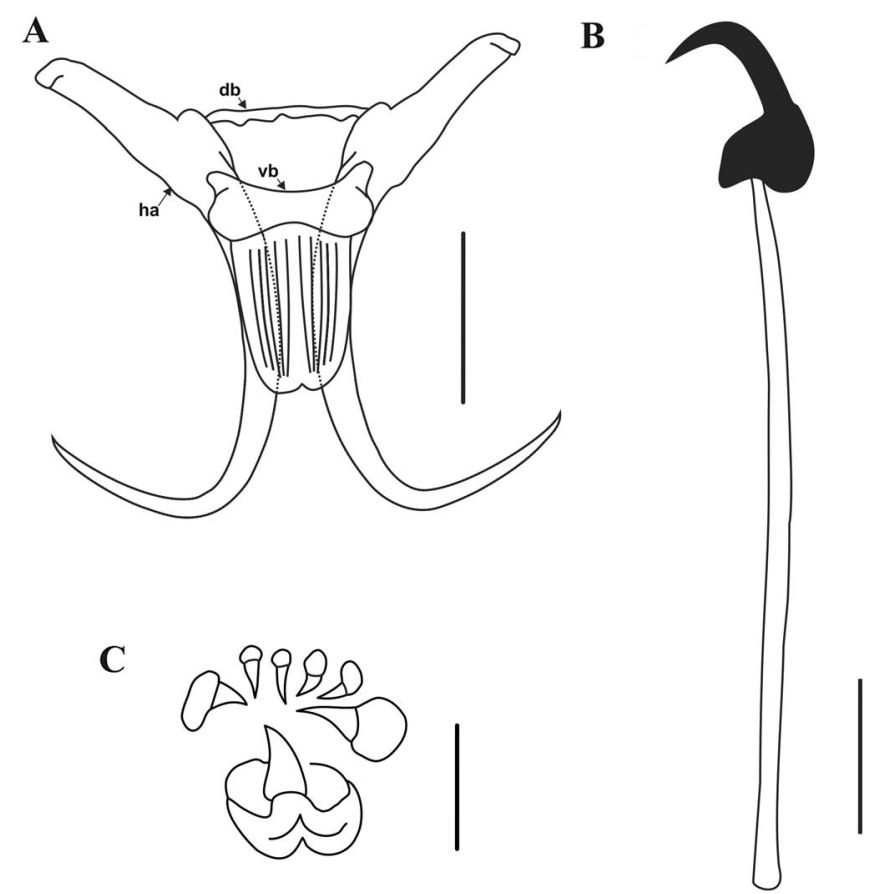

Figure 2. Line drawings of the haptoral sclerites and MCO of Gyrodactylus magadiensis n. sp. from Alcolapia grahami in Lake Magadi, Kenya. (A) Haptoral sclerites with hamulus (ha), dorsal bar (db), and ventral bar (vb); (B) marginal hook; (C) male copulatory organ (MCO). Scale bars - (A) $20 \mu \mathrm{m}$; (B and C) $5 \mu \mathrm{m}$.

beginning of uterus. Pharyngeal bulb 21.1 (13.8-29.7) long, with anterior bulb 19.7 (13.6-28.2) wide and posterior bulb 20.7 (14.7-32.3) wide. Intestinal crura not spreading further than anterior edge of testes. Male copulatory organ (MCO) 11.4 (9.6-13.9) long and 8.5 (6.9-10.2) wide, situated posteriorly to pharyngeal bulb, armed with one central spine and six spinelets (two large and four small) (Figs. 2C, 3G). 

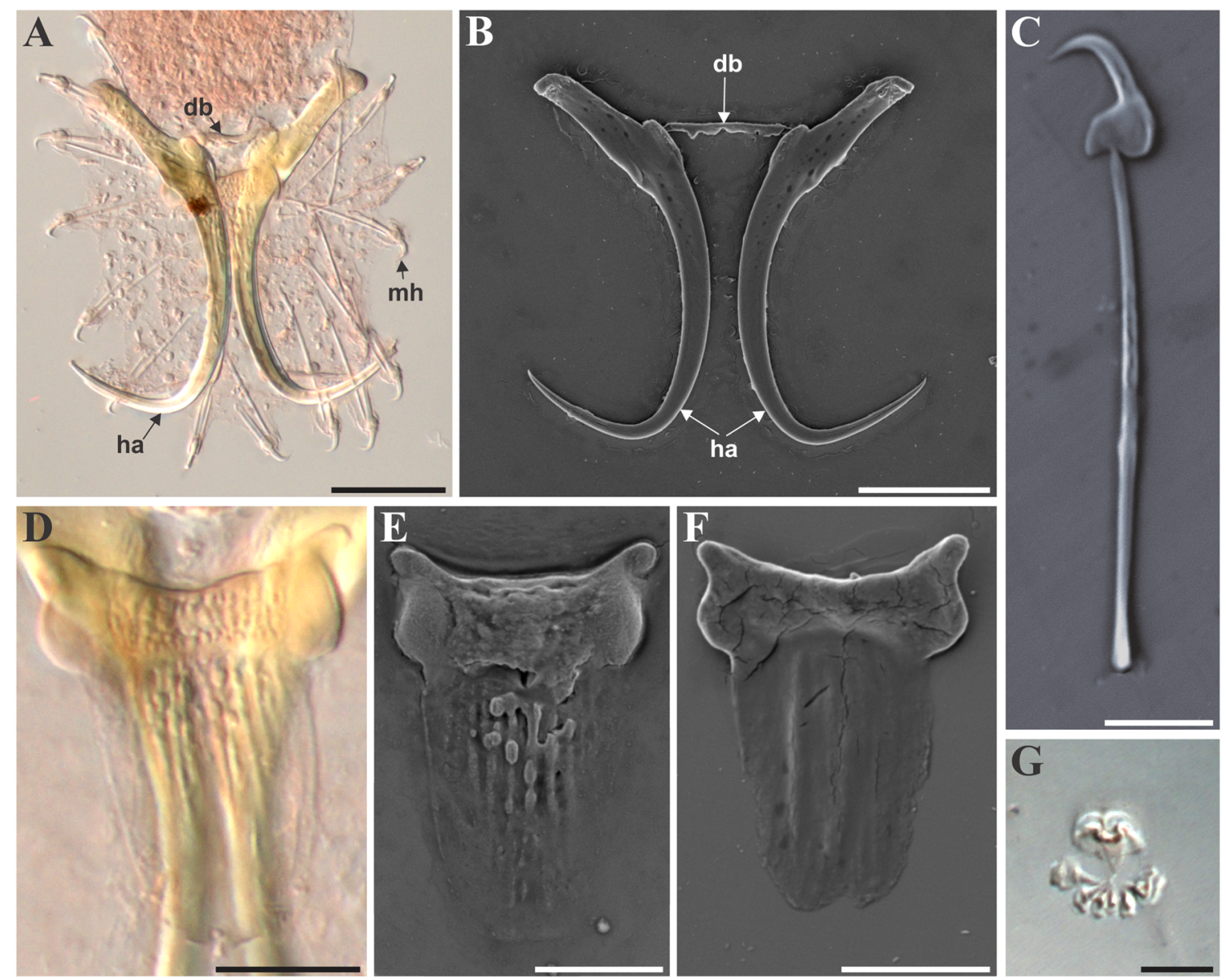

Figure 3. Light (LM) and scanning electron (SEM) micrographs of the haptoral sclerites and male copulatory organ (MCO) of Gyrodactylus magadiensis n. sp. (A) Haptoral sclerites with hamulus (ha), dorsal bar (db), and marginal hooks (mh), GAP (LM); (B) hamulus (ha) and dorsal bar (db) after soft tissue digestion (SEM); (C) isolated marginal hook (SEM); (D) dorsal bar (LM); (E) dorsal view of dorsal bar (SEM); (F) ventral view of dorsal bar (SEM); (G) male copulatory organ (MCO) with large central spine and six spinelets, two large and four small (LM). Scale bars - (A and B) $20 \mu \mathrm{m}$; (C and G) $5 \mu \mathrm{m}$; (D-F) $10 \mu \mathrm{m}$.

Hamuli (Figs. 2A, 3A and B) slender, 62.8 (53.6-73.7) long; shaft even more slender 38.6 (34.8-43.6) long; narrow point $24.2(19.9-29.5)$ long ending in a sharp point. Hamulus not sharply curved, aperture angle $59.9^{\circ}\left(50.1^{\circ}-78.1^{\circ}\right)$; root straight, 22.3 (16.9-29.9) long. Dorsal bar simple, 13 (9.9-17.78) long and 2.6 (2.2-3.6) wide (Figs. 2A, 3A and B). Ventral bar 27.5 (22.6-33.3) long, 20.8 (14.6-26.4) wide; ventral bar processes small, anterolateral, rounded and slightly curved outward, 3 (2-4) long; ventral bar membrane ovoid, tongue-shaped with notch centrally at posterior, 18.7 (15.9-22.8) long (Figs. 2A, 3D-F). Marginal hooks 27.7 (23.4-30) long; hook shaft 23.1 (18.9-26) long with slight rounded swelling terminally (distally); marginal hook sickle slightly curved and tilted forward, 5.4 (4.7-6.1) long; point long and continuously curved from sickle, 4 (3-4.7) wide and distal width 3.4 (2.9-4) (Figs. 2B, 3C). Heel rounded strongly toward shaft; toe trapezoidal to square $1.8(1.5-2.2)$ long, with sharp indentation on inferior edge between toe tip and shaft attachment point; long bridge prior to reaching marginal sickle shaft; long lateral edge of toe. Marginal hook aperture 4.4 (3.6-5.1); hook instep height 0.8 (0.62-1); filament loop 8 (6.5-9.2) long.

\section{Molecular identity of Gyrodactylus magadiensis $\mathbf{n}$. sp.}

All 10 specimens produced identical sequence data for the ITS fragment analysed. The amplified region was $882 \mathrm{bp}$ in length, with the size of the 18S, ITS1, 5.8S, ITS2, and 28S rDNA fragments $24,385,158,291$, and 24 bp long, respectively. Alignment of the sequence to other data retrieved from GenBank produced a 1088 bp alignment, of which 636 positions were conserved, 446 variable, and 399 parsimony informative. Gyrodactylus magadiensis $\mathrm{n}$. sp. was only distantly related to most other Gyrodactylus species (Table 4), most closely to Gyrodactylus branchicus Malmberg, 1964 (23.2\%) and most distantly to Gyrodactylus katamba García-Vásquez, Guzmán-Valdivieso, Razo-Mendivil, and Rubio-Godoy 2018 (25.4\%). Distances of $0.45-25.4 \%$ were observed between species included in these analyses, while intraspecific distances of $0.00-1.14 \%$ were seen. The latter would suggest that taxa with more than $1.14 \%$ sequence divergence are distinct species, indicating that sequences for distinct species with less than that (in this case G. katamba and Gyrodactylus lamothei 
Table 4. Sequence divergence (\%) based on average uncorrected p-distance separating Gyrodactylus magadiensis n. sp. (values in bold) from other Gyrodactylus species. Intraspecific distances indicated by shaded cells.

\begin{tabular}{|c|c|c|c|c|c|c|c|c|c|c|c|c|c|c|c|c|c|c|c|c|c|c|c|}
\hline Species & Accession & & 1 & 2 & 3 & 4 & 5 & 6 & 7 & 8 & 9 & 10 & 11 & 12 & 13 & 14 & 15 & 16 & 17 & 18 & 19 & 20 & 21 \\
\hline G. magadiensis $\mathrm{n} . \mathrm{sp}$. & MN738699 & 1 & - & & & & & & & & & & & & & & & & & & & & \\
\hline G. branchicus & AY061977 & 2 & 23.20 & - & & & & & & & & & & & & & & & & & & & \\
\hline G. branchicus & AF156669 & 3 & 23.44 & 0.31 & - & & & & & & & & & & & & & & & & & & \\
\hline G. rarus & AY061976 & 4 & 23.32 & 0.73 & 1.05 & - & & & & & & & & & & & & & & & & & \\
\hline G. rarus & FJ435193 & 5 & 23.57 & 0.73 & 1.05 & 0.21 & - & & & & & & & & & & & & & & & & \\
\hline G. rarus & AY338445 & 6 & 24.28 & 0.75 & 1.07 & 0.21 & 0.00 & - & & & & & & & & & & & & & & & \\
\hline G. perlucidus & FJ435202 & 7 & 23.55 & 14.91 & 14.91 & 14.91 & 15.13 & 15.53 & - & & & & & & & & & & & & & & \\
\hline G. medaka & LC368478 & 8 & 23.54 & 12.18 & 12.08 & 12.08 & 12.08 & 12.39 & 16.96 & - & & & & & & & & & & & & & \\
\hline G. medaka & LC368475 & 9 & 23.66 & 12.61 & 12.50 & 12.50 & 12.50 & 12.83 & 17.07 & 1.14 & - & & & & & & & & & & & & \\
\hline G. medaka & LC368479 & 10 & 23.78 & 12.71 & 12.61 & 12.61 & 12.61 & 12.93 & 17.18 & 1.14 & 0.21 & - & & & & & & & & & & & \\
\hline G. medaka & LC368477 & 11 & 23.78 & 12.71 & 12.61 & 12.61 & 12.61 & 12.93 & 17.18 & 1.04 & 0.10 & 0.10 & - & & & & & & & & & & \\
\hline G. alexgusevi & AY061979 & 12 & 24.04 & 9.64 & 9.96 & 9.64 & 9.64 & 9.89 & 17.63 & 14.16 & 14.38 & 14.48 & 14.48 & - & & & & & & & & & \\
\hline G. groenlandicus & KJ095104 & 13 & 24.03 & 15.77 & 15.88 & 15.88 & 15.88 & 16.25 & 8.67 & 17.26 & 17.26 & 17.26 & 17.36 & 18.58 & - & & & & & & & & \\
\hline G. alexanderi & JN695633 & 14 & 24.16 & 7.25 & 7.35 & 7.14 & 7.14 & 7.33 & 16.20 & 12.93 & 13.25 & 13.14 & 13.14 & 10.98 & 17.54 & - & & & & & & & \\
\hline G. alexanderi & JF836144 & 15 & 24.16 & 7.14 & 7.25 & 7.04 & 7.04 & 7.22 & 16.09 & 13.04 & 13.35 & 13.25 & 13.25 & 10.88 & 17.36 & 0.10 & - & & & & & & \\
\hline G. alexanderi & FJ435201 & 16 & 24.27 & 7.15 & 7.26 & 7.05 & 7.05 & 7.23 & 16.11 & 13.07 & 13.39 & 13.28 & 13.28 & 10.89 & 17.65 & 0.00 & 0.00 & - & & & & & \\
\hline G. wilkesi & LT719091 & 17 & 24.97 & 15.59 & 15.71 & 15.71 & 15.71 & 15.71 & 8.99 & 18.57 & 18.80 & 18.80 & 18.91 & 18.66 & 5.98 & 17.13 & 17.13 & 17.04 & - & & & & \\
\hline G. lamothei & KX555668 & 18 & 25.23 & 22.98 & 23.09 & 22.86 & 23.09 & 23.09 & 22.70 & 23.22 & 22.99 & 22.99 & 22.99 & 22.44 & 23.76 & 22.06 & 22.17 & 22.08 & 23.22 & - & & & \\
\hline G. lamothei & KX555666 & 19 & 25.30 & 23.05 & 23.17 & 22.94 & 23.17 & 23.17 & 22.79 & 23.29 & 23.06 & 23.06 & 23.06 & 22.51 & 23.74 & 22.13 & 22.25 & 22.16 & 23.20 & 0.00 & - & & \\
\hline G. lamothei & KX555667 & 20 & 25.33 & 23.08 & 23.19 & 22.96 & 23.19 & 23.19 & 22.82 & 23.32 & 23.09 & 23.09 & 23.09 & 22.54 & 23.77 & 22.16 & 22.27 & 22.18 & 23.23 & 0.00 & 0.00 & - & \\
\hline G. katamba & KR815854 & 21 & 25.36 & 23.00 & 23.11 & 22.88 & 23.11 & 23.14 & 22.97 & 23.12 & 22.89 & 22.89 & 22.89 & 22.46 & 24.03 & 22.08 & 22.20 & 22.11 & 23.29 & 0.45 & 0.56 & 0.56 & - \\
\hline
\end{tabular}




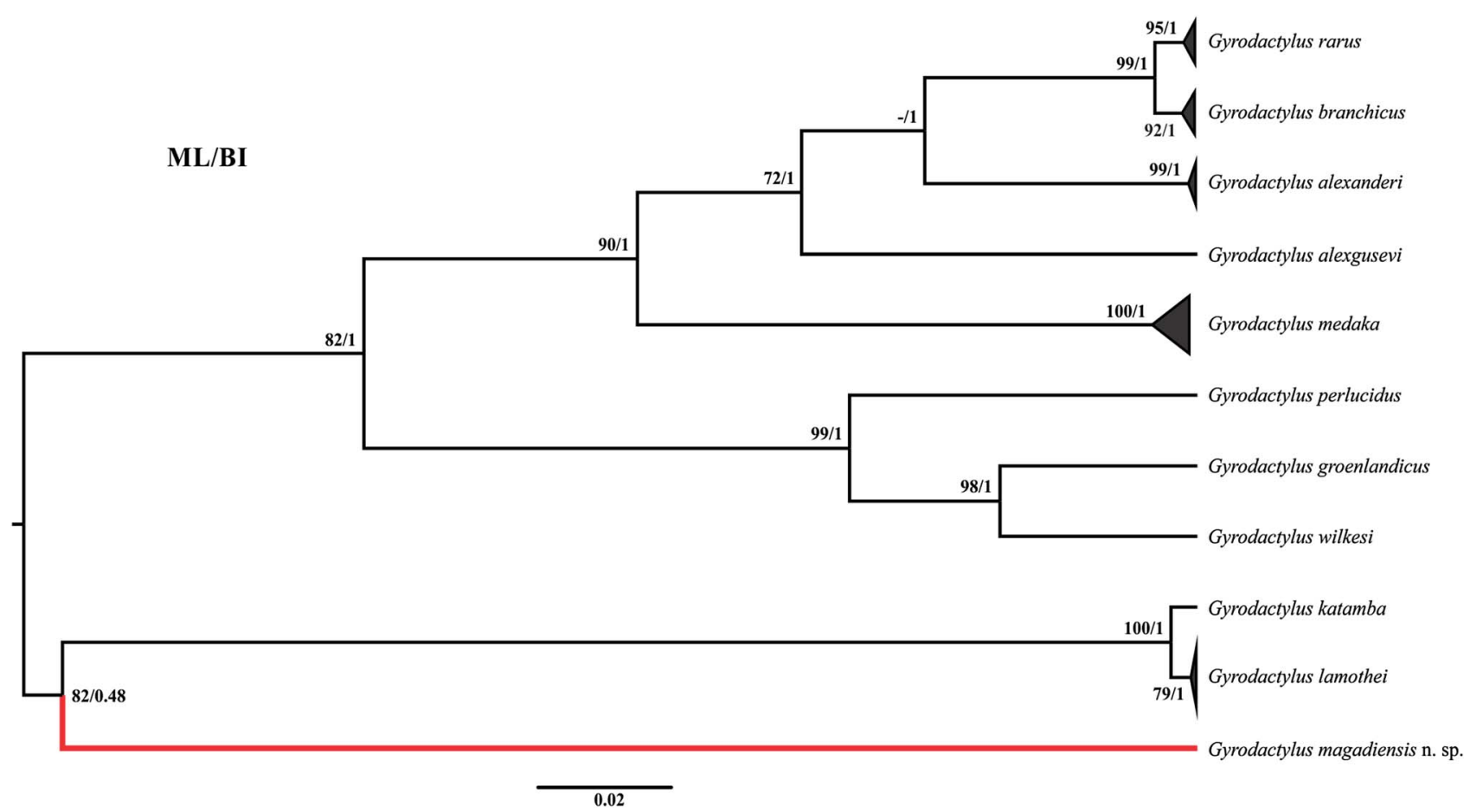

Figure 4. Evolutionary history of Gyrodactylus magadiensis n. sp. based on Bayesian Inference approaches using ITS sequences for selected gyrodactylids. Statistical support for Bayesian inference (BI) and maximum likelihood (ML) methods indicated at branch nodes with posterior probabilities and bootstrap support indicated, respectively (ML/BI).

Mendoza-Palmero, Sereno-Uribe and Salgado-Maldonado, 2009, and G. branchicus and G. rarus Wagener, 1910) need to be revised to produce a robust criteria to identify species based on ITS rDNA. Topologies of phylogenetic analyses based on ML and BI methods produced similar results, thus a single combined tree is shown in Figure 4. In all cases, G. magadiensis n. sp. formed a distinct, well supported linage from its congeners. Gyrodactylus magadiensis n. sp. appeared to be most closely related to a clade of G. katamba and G. lamothei in all cases.

\section{Differential diagnosis}

In comparison to other Gyrodactylus species described from African cichlid fishes, the marginal hooks of G. magadiensis n. sp. are most similar to those of Gyrodactylus cichlidarum Paperna, 1968, Gyrodactylus yacatli García-Vásquez, Hansen, Christison, Bron and Shinn, 2011 and Gyrodactylus ulinganisus García-Vásquez, Hansen, Christison, Bron and Shinn, 2011 in that the sickle is smoothly curved (except $G$. yacatli) and the toe is almost square. The marginal hooks of the new species can be distinguished from these species in that the toe is more pronounced than in $G$. cichlidarum and $G$. ulinganisus; the indentation on inferior edge between the toe tip and shaft attachment point is more pronounced than in G. cichlidarum and $G$. ulinganisus; the bridge prior to reaching the marginal sickle shaft is longer than in G. cichlidarum and G. ulinganisus; the lateral edge of the toe is longer than in G. yacatli; and the sickle is angled forward (similar only to G. yacatli). The heel of G. magadiensis n. sp. is also notably rounded, only slightly similar to that of Gyrodactylus thysi Vanhove, Snoeks, Volckaert and Huyse, 2011, Gyrodactylus thlapi Christison,
Shinn and van As, 2005 and the illustration of Gyrodactylus niloticus Cone, Arthur and Bondad-Reantaso, 1995 by Cone et al. [9] (junior synonym of G. cichlidarum [13]).

In terms of the ventral bar, G. magadiensis n. sp. can be differentiated from other Gyrodactylus species infecting African cichlids based on the distinct tongue shape of the membrane, medial notch in posterior of membrane, rounded lateral ends of the bar itself, and anterolateral processes rounded and slightly curved outward. These features are most similar to the ventral bar of $G$. cichlidarum, but specifically the shape of the membrane can easily distinguish these species. The long and narrow nature of the hamuli are reminiscent of those of G. malalai, Gyrodactylus ergensi Přikrylová, Matějusová, Musilová and Gelnar, 2009 and Gyrodactylus nyanzae Paperna, 1973. However, the hamuli of $G$. magadiensis n. sp. can be distinguished by the lack of an indentation of the root above the attachment of the dorsal bar as in G. malalai and G. ergensi, and the more robust root in comparison to $G$. nyanzae. The MCO of G. magadiensis n. sp. has six spinelets, whereas most of the other species for African cichlids have 4, 5 or 7 (with the exception of Gyrodactylus shariffi Cone, Arthur and BondadReantaso, 1995 and G. cichlidarum which can have six).

\section{Discussion}

Gyrodactylus magadiensis n. sp. is the second record of a Gyrodactylus species infecting a cichlid from Kenya. However, this is the first gyrodactylid to be described from the extreme conditions of Lake Magadi and from Alcolapia grahami. The ability of G. magadiensis n. sp. to persist and thrive in the extreme condition of Lake Magadi is truly impressive. 
Although G. salinae survives in the extreme salinity and temperature of Cervia Saline in Italy [46], this species is genetically very distant from $G$. magadiensis n. sp. and the morphologies of the species do not share many similarities. It is thus unlikely that these species, which are both able to survive extreme conditions, originated from the same lineage, indicating that this adaptation has occurred at least twice convergently.

Based on both morphology and genetic data, the material studied here is markedly distinct from all other information available for African gyrodactylid monogeneans. Regarding the genetic identity of G. magadiensis n. sp., a gyrodactylid infecting a marine Gasterosteiformes host (Gasterosteidae) collected in Finland and Belgium is the most closely related (23.2\%) based on uncorrected $p$-distances, while none of the sequences for Gyrodactylus species from African cichlids were identified as close relatives using BLASTn, even though most of these African species have representative sequence data. In fact, it would appear that most of the species identified as close relatives to G. magadiensis n. sp. are marine species, which is puzzling. However, as can be seen from the topology of the phylogenetic analyses shown in Figure 4, G. magadiensis n. sp. groups with a clade of G. lamothei and G. katamba, both species from freshwater systems in Mexico. This association is only weakly supported by BI analyses (0.48), but more strongly by $\mathrm{ML}$ approaches $(82 \%)$.

\section{Conclusion}

Gyrodactylus magadiensis n. sp. is described here on the basis of its morphology and genetic identity. The species can be distinguished from congeners parasitising other African cichlids based on the comparatively long and narrow hamuli, a ventral bar with small rounded anterolateral processes and a tongue-shaped posterior membrane, and marginal hooks with slender sickles which are angled forward, a trapezoid to square toe, rounded heel, a long bridge prior to reaching the marginal sickle shaft, and a long lateral edge of the toe. Genetically, this species is distinct from all other monogenean species, with more than $23.2 \%$ pairwise divergence between it and its closest relatives.

\section{Conflict of interest}

The authors state that there is no conflict of interest

Acknowledgements. The authors would like to thank the University of Johannesburg for the use of SPECTRUM and funding from the University of Johannesburg Research Committee (AA-O). JNM and AA-O thank the National Research Foundation (NRF) of South Africa for their financial support. Funding to QMDS was provided through the Global Excellence and Stature Postdoctoral Fellowship at the University of Johannesburg. We thank Grace Moyo for her contributions to an earlier draft.

\section{References}

1. Altschul SF, Gish W, Miller W, Myers EW, Lipman DJ. 1990. Basic local alignment search tool. Journal of Molecular Biology, 215, 403-410.
2. Arthur JR, Te BQ. 2006. Checklist of the parasites of fishes of Viet Nam. FAO Fisheries Technical Paper, 369. Rome, Italy: FAO, 1-133.

3. Avenant-Oldewage A, le Roux LE, Mashego SN, van Vuuren BJ. 2014. Paradiplozoon ichthyoxanthon n. sp. (Monogenea: Diplozoidae) from Labeobarbus aeneus (Cyprinidae) in the Vaal River, South Africa. Journal of Helminthology, 88, 166-172.

4. Bakke TA, Cable J, Harris PD. 2007. The biology of gyrodactylid monogeneans: the "Russian-Doll Killers". Advances in Parasitology, 64, 161-460.

5. Bergman AN, Laurent P, Otiang'a-Owiti G, Bergman HL, Walsh PJ, Wilson P, Wood CM. 2003. Physiological adaptations of the gut in the Lake Magadi tilapia, Alcolapia grahami, an alkaline- and saline-adapted teleost fish. Comparative Biochemistry and Physiology A - Molecular and Integrative Physiology, 136, 701-715.

6. Bouckaert R, Heled J, Kühnert D, Vaughan T, Wu CH, Xie D, Suchard MA, Rambaut A, Drummond AJ. 2014. BEAST 2: a software platform for Bayesian evolutionary analysis. PLoS Computational Biology, 10, e1003537.

7. Butzer KW, Isaac GL, Richardson JL, Washbourn-kamau C. 1972. Radiocarbon dating of east african lake levels. Science, 175, 1069-1076.

8. Christison KW, Shinn AP, Van As JG. 2005. Gyrodactylus thlapi n. sp. (Monogenea) from Pseudocrenilabrus philander philander (Weber) (Cichlidae) in the Okavango Delta, Botswana. Systematic Parasitology, 60, 165-173.

9. Cone DK, Arthur JR, Bondad-Reantaso MG. 1995. Description of two new species of Gyrodactylus von Nordmann, 1832 (Monogenea) from cultured Nile Tilapia, Tilapia nilotica (Cichlidae), in the Philippines. Journal of the Helminthological Society of Washington, 62, 6-9.

10. Dos Santos QMD, Avenant-Oldewage A. 2015. Soft tissue digestion of Paradiplozoon vaalense for SEM of sclerites and simultaneous molecular analysis. Journal of Parasitology, 101, 94-97.

11. Dos Santos QMD, Dzika E, Avenant-Oldewage A. 2019. Using scanning electron microscopy (SEM) to study morphology and morphometry of the isolated haptoral sclerites of three distinct diplozoid species. PLoS One, 14, 1-24.

12. El-Naggar AM, El-Tantawy SA. 2003. The dynamics of gill monogenean communities on cichlid fish hosts inhabiting Damietta branch of the river Nile: long-term changes in species richness and community structure. Journal of the Egyptian German Society of Zoology, 41D, 187-220.

13. Ergens R. 1969. The suitability of ammonium picrate-glycerin in preparing slides of lower Monogenoidea. Folia Parasitologica, 16,320 .

14. Eugster HP. 1980. Lake Magadi, Kenya, and its precursors, in Developments in sedimentology. Hypersaline brines evaporitic environments. Nissenbaum A, Editor. Elsevier: Amsterdam, Netherlands. p. 195-232.

15. García-Vásquez A, Hansen H, Shinn AP. 2007. A revised description of Gyrodactylus cichlidarum Paperna, 1968 (Gyrodactylidae) from the Nile tilapia, Oreochromis niloticus niloticus (Cichlidae), and its synonymy with G. niloticus Cone, Arthur et Bondad-Reantaso, 1995. Folia Parasitologica, 54, 129-140.

16. García-Vásquez A, Hansen H, Christison KW, Bron JE, Shinn AP. 2011. Description of three new species of Gyrodactylus von Nordmann, 1832 (Monogenea) parasitising Oreochromis niloticus niloticus (L.) and O. mossambicus (Peters) (Cichlidae). Acta Parasitologica, 56, 20-33.

17. García-Vásquez A, Razo-Mendivil U, Rubio-Godoy M. 2015. Morphological and molecular description of eight new species of Gyrodactylus von Nordmann, 1832 (Platyhelminthes: 
Monogenea) from poeciliid fishes, collected in their natural distribution range in the Gulf of Mexico slope, Mexico. Parasitology Research, 114, 3337-3355.

18. García-Vásquez A, Guzmán-Valdivieso I, Razo-Mendivil U, Rubio-Godoy M. 2017. Three new species of Gyrodactylus von Nordmann, 1832 described from Goodea atripinnis (Pisces: Goodeidae), an endemic freshwater fish from the central highlands of Mexico. Parasitology Research, 117, 139-150.

19. García-Vásquez A, Razo-Mendivil U, Rubio-Godoy M. 2017. Triple trouble? Invasive poeciliid fishes carry the introduced tilapia pathogen Gyrodactylus cichlidarum in the Mexican highlands. Veterinary Parasitology, 235, 37-40.

20. García-Vásquez A, Pinacho-Pinacho CD, Guzmán-Valdivieso I, Salgado-Maldonado G, Rubio-Godoy M. 2019. New species of Gyrodactylus von Nordmann, 1832 from native fish from Chiapas, Mexico, studied by morphology and molecular analyses. Acta Parasitologica, 64, 551-565.

21. Gilmore SR, Cone DK, Lowe G, King SK, Jones SRM, Abbott CL. 2012. Molecular phylogeny of Gyrodactylus (monogenea) parasitizing fishes in fresh water, estuarine, and marine habitats in Canada. Canadian Journal of Zoology, 90, 776-786.

22. Goetz C, Hillaire-Marcel C. 1992. U-series disequilibria in early diagenetic minerals from Lake Magadi sediments, Kenya: dating potential. Geochimica et Cosmochimica Acta, 56, 1331-1341.

23. Hansen H, Jørgensen A, Mo TA. 2012. Spin-off from routine parasite diagnostics of Atlantic salmon; first report of Gyrodactylus alexanderi in Norway. Bulletin of the European Association of Fish Pathologists, 32, 14-18.

24. Harris PD, Shinn AP, Cable J, Bakke TA. 2004. Nominal species of the genus Gyrodactylus von Nordmann 1832 (Monogenea: Gyrodactylidae), with a list of principal host species. Systematic Parasitology, 59, 1-27.

25. Heglasová I, Nezhybová V, Přikrylová I. 2018. An amended description of two Gyrodactylus species (Platyhelminthes: Monogenea) parasitizing Antarctic Notothenioid fish. Journal of Helminthology, 97, 1-9.

26. Huyse T, Volckaert FAM. 2002. Identification of a hostassociated species complex using molecular and morphometric analyses, with the description of Gyrodactylus rugiensoides $\mathrm{n}$. sp. (Gyrodactylidae, Monogenea). International Journal for Parasitology, 32, 907-919.

27. Huyse T, Audenaert V, Volckaert FAM. 2003. Speciation and host-parasite relationships in the parasite genus Gyrodactylus (Monogenea, Platyhelminthes) infecting gobies of the genus Pomatoschistus (Gobiidae, Teleostei). International Journal for Parasitology, 33, 1679-1689.

28. Jones BF, Eugster HP, Retting SL. 1977. Hydrochemistry of the Lake Magadi basin, Kenya. Geochemica et Cosmochimica Acta, 41, 53-72.

29. Jorissen MWP, Pariselle A, Huyse T, Vreven EJ, Kasembele GK, Artois T, Vanhove MPM. 2018. Diversity and host specificity of monogenean gill parasites (Platyhelminthes) of cichlid fishes in the Bangweulu-Mweru ecoregion. Journal of Helminthology, 92, 417-437.

30. Katoh K, Standley DM. 2013. MAFFT multiple sequence alignment software version 7: improvements in performance and usability. Molecular Biology and Evolution, 30, 772-780.

31. Katoh K, Misawa K, Kuma K, Miyata T. 2002. MAFFT: a novel method for rapid multiple sequence alignment based on fast Fourier transform. Nucleic Acids Research, 30, 3059-3066.

32. Kearse M, Moir R, Wilson A, Stones-Havas S, Cheung M, Sturrock S, Buxton S, Cooper A, Markowitz S, Duran C, Thierer T, Ashton B, Meintjes P, Drummond A. 2012. Geneious basic: an integrated and extendable desktop software platform for the organization and analysis of sequence data. Bioinformatics, 28, 1647-1649.
33. King SD, Cone DK, Gilmore SR, Jones SRM, Abbott CL. 2014. Supplemental description and phylogenetic placement of Gyrodactylus corti (Monogenea: Gyrodactylidae) parasitizing captive wolf-eel Anarrhichthys ocellatus in British Columbia. Comparative Parasitology, 81, 225-231.

34. Kumar S, Stecher G, Tamura K. 2016. MEGA7: molecular evolutionary genetics analysis version 7.0 for bigger datasets. Molecular Biology and Evolution, 33, 1870-1874.

35. Luo HY, Nie P, Zhang YA, Wang GT, Yao WJ. 2002. Molecular variation of Bothriocephalus acheilognathi Yamaguti, 1934 (Cestoda: Pseudophyllidea) in different fish host species based on ITS rDNA sequences. Systematic Parasitology, 52, 159-166.

36. Luton K, Walker D, Blair D. 1992. Comparisons of ribosomal internal transcribed spacers from two congeneric species of flukes (Platyhelminthes: Trematoda: Digenea). Molecular and Biochemical Parasitology, 56, 323-327.

37. Maillard C, Gonzalez J, Noisy D. 1982. A scanning electron microscope study of the male copulatory sclerite of the monogenean Diplectanum aequans. Parasitology, 84, 63-64.

38. Maina JN. 1990. A study of the morphology of the gills of an extreme alkalinity and hyperosmotic adapted teleost Oreochromis alcalicus grahami (Boulenger) with particular emphasis on the ultrastructure of the chloride cells and their modifications with water dilut. Anatomy and Embryology, 181, 83-98.

39. Maina JN, Kisia SM, Wood CM, Narahara AB, Bergman HL, Laurent P, Walsh PJ. 1996. A comparative allometric study of the morphometry of the gills of an alkalinity adapted cichlid fish, Oreochromis alcalicus grahami, of Lake Magadi, Kenya. International Journal of Salt Lake Research, 5, 131-156.

40. Maina JN, Wood CM, Narahara A, Bergman HL, Laurent P, Walsh PJ. 1996. Morphology of the swim (air) bladder of a cichlid teleost: Oreochromis alcalicus grahami (Trewavas, 1983), a fish adapted to a hyperosmotic, alkaline, and hypoxic environment: a brief outline of the structure and function of the swimbladder, in Fish Morphology, Mushi JS, Dutta HM, Editors. 1st edn., Taylor \& Francis: London. p. 179-192.

41. Maina JN, Kavembe GD, Papah MB, Mashiteng R, Wood CM, Bianchini A, Bianchini LF, Bergman HL, Johannsson OE, Laurent P, Chevalier C, Ojoo RO. 2019. Sizes, condition factors and sex ratios of the scattered populations of the small cichlid fish, Alcolapia grahami, that inhabits the lagoons and sites of Lake Magadi (Kenya), one of the most extreme aquatic habitat on Earth. Environmental Biology of Fishes, 102, 1265-1280.

42. Malmberg G. 1957. Om förekomsten av Gyrodactylus på svenska fiskar (In Swedish.). Skrifter Utgivna av Södra Sveriges Fiskeriförening, Årsskrift, 1956, 19-76.

43. Ministry of Agriculture Fisheries and Food, Great Britain. 1986. Manual of veterinary parasitological laboratory techniques. Richmond, UK: Her Majesty's Stationery Office. 160 p.

44. Mo TA, Appleby C. 1990. A special technique for studying haptoral sclerites of monogeneans. Systematic Parasitology, 17, 103-108.

45. Mousavi HE, Omidzahir S, Soltani M, Shayan P, Ebrahimzadeh E, Mousavi S, Hoseini M. 2013. Morphometrical and molecular characterization of Gyrodactylus cichlidarum (Gyrodactylidae) from Astronotus ocellatus (Cichlidae) in Iran. Comparative Clinical Pathology, 22, 1093-1097.

46. Narahara A, Bergman HL, Laurent P, Maina JN, Walsh PJ, Wood CM. 1996. Respiratory physiology of the Lake Magadi tilapia (Oreochromis alcalicus grahami), a fish adapted to a hot, alkaline, and frequently hypoxic environment. Physiological Zoology, 69, 1114-1136.

47. Nitta M, Nagasawa K. 2018. Gyrodactylus medaka n. sp. (Monogenea: Gyrodactylidae) parasitic on wild and laboratoryreared medaka Oryzias latipes (Beloniformes: Adrianichthyidae) in Japan. Parasitology International, 67, 651-658. 
48. Paladini G, Huyse T, Shinn AP. 2011. Gyrodactylus salinae n. sp. (Platyhelminthes: Monogenea) infecting the south European toothcarp Aphanius fasciatus (Valenciennes) (Teleostei, Cyprinodontidae) from a hypersaline environment in Italy. Parasites and Vectors, 4, 1-12.

49. Paperna I. 1968. Monogenetic trematodes collected from fresh water fish in Ghana. Second report. Bamidgeh, 20, 88-99.

50. Paperna I. 1973. New species of Monogenea (Vermes) from African freshwater fish. A preliminary report. Revue de Zoologie et de Botanique Africaines, 87, 505-518.

51. Paperna I. 1979. Monogenea of inland water fish in Africa. Annales du Musée Royal de l'Afrique Centrale, Science Zoologique, 226, 1-131.

52. Parkinson J. 1914. The East African Trough in the neighbourhood of the Soda Lakes. The Geographical Journal, 44, 33-46.

53. Pörtner HO, Schulte PM, Wood CM, Schiemer F. 2010. Niche dimensions in fishes: an integrative view. Physiological and Biochemical Zoology, 83, 808-826.

54. Přikrylová I, Matějusová I, Musilová N, Gelnar M. 2009. Gyrodactylus species (Monogenea: Gyrodactylidae) on the cichlid fishes of Senegal, with the description of Gyrodactylus ergensi $\mathrm{n}$. sp. from Mango tilapia, Sarotherodon galilaeus L. (Teleostei: Cichilidae). Parasitology Research, 106, 1-6.

55. Přikrylová I, Blažek R, Gelnar M. 2012. Gyrodactylus malalai sp. nov. (Monogenea, Gyrodactylidae) from Nile tilapia, Oreochromis niloticus (L.) and Redbelly tilapia, Tilapia zillii (Gervais) (Teleostei, Cichlidae) in the Lake Turkana, Kenya. Acta Parasitologica, 57, 122-130.

56. Randall DJ, Wood CM, Perry SF, Bergman H, Maloiy GMO, Mommsen TP, Wright PA. 1989. Urea excretion as a strategy for survival in a fish living in a very alkaline environment. Nature, 337, 165-166.

57. Řehulková E, Seifertová M, Přikrylová I, Francová K. 2018. Monogenea, in Guide to Parasites African Freshw. Fishes Divers. Ecol. Res. Methods, Scholz T, Vanhove MPM, Smit N, Jayasundera Z, Gelnar M, Editors. ABC Taxa: CEBioS, Royal Belgian Institute of Natural Sciences: Brussels, Belgium. p. $185-243$.

58. Rokicka M, Lumme J, Zieôtara MS. 2009. Two new Antarctic Gyrodactylus Species (Monogenoidea): description and phylogenetic characterization. Journal of Parasitology, 95, 1112-1119.

59. Rubio-Godoy M, Razo-Mendivil U, García-Vásquez A, Freeman MA, Shinn AP, Paladini G. 2016. To each his own: no evidence of gyrodactylid parasite host switches from invasive poeciliid fishes to Goodea atripinnis Jordan (Cyprinodontiformes: Goodeidae), the most dominant endemic freshwater goodeid fish in the Mexican Highlands. Parasites \& Vectors, 9, 1-21.

60. Shinn AP, Hansen H, Olstad K, Bachmann L, Bakke TA. 2004. The use of morphometric characters to discriminate specimens of laboratory-reared and wild populations of Gyrodactylus salaris and G. thymalli (Monogenea). Folia Parasitologica, 51, 239-252.
61. Talling JF, Talling IB. 1965. The chemical composition of African Lake water. Internationale Revue Der Gesamten Hydrobiologie Und Hydrographie, 50, 421-463.

62. Trewavas E. 1983. Tilapiine fishes of the Genera Sarotherodon, Oreochromis and Danakilia. London: Trustees of the British Museum (Natural History).

63. Truter M, Přikrylová I, Malherbe W, Smit NJ. 2016. First report of metazoan parasites from the cichlid Pseudocrenilabrus philander and the cyprinid Enteromius paludinosus in a South African Ramsar wetland. African Journal of Aquatic Science, 41, 499-503.

64. Vanhove MPM, Snoeks J, Volckaert FAM, Huyse T. 2011. First description of monogenean parasites in Lake Tanganyika: The cichlid Simochromis diagramma (Teleostei, Cichlidae) harbours a high diversity of Gyrodactylus species (Platyhelminthes, Monogenea). Parasitology, 138, 364-380.

65. Vanhove MPM, Briscoe AG, Jorissen MWP, Littlewood DTJ, Huyse T. 2018. The first next-generation sequencing approach to the mitochondrial phylogeny of African monogenean parasites (Platyhelminthes: Gyrodactylidae and Dactylogyridae). BMC Genomics, 19, 520.

66. Walsh PJ, Grosell M, Goss GG, Bergman HL, Bergman AN, Wilson P, Laurent P, Alper SL, Smith CP, Kamunde C, Wood CM. 2001. Physiological and molecular characterization of urea transport by the gills of the Lake Magadi tilapia (Alcolapia grahami). Journal of Experimental Biology, 204, 509-520.

67. Wilson PJ, Wood CM, Maina JN, White BN. 2000. Genetic structure of Lake Magadi tilapia populations. Journal of Fish Biology, 56, 590-603.

68. Wood CM, Perry SF, Wright PA, Bergman HL, Randall DJ. 1989. Ammonia and urea dynamics in the Lake Magadi tilapia, a ureotelic teleost fish adapted to an extremely alkaline environment. Respiration Physiology, 77, 1-20.

69. Wood CM, Bergman HL, Laurent P, Maina JN, Narahara A, Walsh PJ. 1994. Urea production, acid-base regulation and their interactions in the Lake Magadi tilapia, a unique teleost adapted to a highly alkaline environment. Journal of Experimental Biology, 189, 13-36.

70. Zahradníčková P, Barson M, Luus-Powell WJ, Přikrylová I. 2016. Species of Gyrodactylus von Nordmann, 1832 (Platyhelminthes: Monogenea) from cichlids from Zambezi and Limpopo river basins in Zimbabwe and South Africa: evidence for unexplored species richness. Systematic Parasitology, 93, 679-700.

71. Zieôtara MS, Lumme J. 2003. The crossroads of molecular, typological and biological species concepts: two new species of Gyrodactylus Nordmann, 1832 (Monogenea: Gyrodactylidae). Systematic Parasitology, 55, 39-52.

72. Zieôtara MS, Arndt A, Geets A, Hellemans B, Volckaert FAM. 2000. The nuclear rRNA region of Gyrodactylus arcuatus and G. branchicus. Journal of Parasitology, 86, 1368-1373.

Cite this article as: Dos Santos QM. Maina JN \& Avenant-Oldewage A. 2019. Gyrodactylus magadiensis n. sp. (Monogenea, Gyrodactylidae) parasitising the gills of Alcolapia grahami (Perciformes, Cichlidae), a fish inhabiting the extreme environment of Lake Magadi, Kenya. Parasite 26, 76. 
Reviews, articles and short notes may be submitted. Fields include, but are not limited to: general, medical and veterinary parasitology; morphology, including ultrastructure; parasite systematics, including entomology, acarology, helminthology and protistology, and molecular analyses; molecular biology and biochemistry; immunology of parasitic diseases; host-parasite relationships; ecology and life history of parasites; epidemiology; therapeutics; new diagnostic tools.

All papers in Parasite are published in English. Manuscripts should have a broad interest and must not have been published or submitted elsewhere. No limit is imposed on the length of manuscripts.

Parasite (open-access) continues Parasite (print and online editions, 1994-2012) and Annales de Parasitologie Humaine et Comparée (1923-1993) and is the official journal of the Sociéte Française de Parasitologie. 